\title{
BMJ Open Role of probiotics in patients with colorectal cancer: a systematic review protocol of randomised controlled trial studies
}

\author{
Ifeoma Julieth Dikeocha (10 , ${ }^{1}$ Abdelkodose Mohammed Al-Kabsi, ${ }^{1}$ \\ Salasawati Hussin, ${ }^{1}$ Mohammed Abdullah Alshawsh (iD ${ }^{2}$
}

To cite: Dikeocha IJ, AlKabsi AM, Hussin S, et al. Role of probiotics in patients with colorectal cancer: a systematic review protocol of randomised controlled trial studies. BMJ Open 2020;10:e038128. doi:10.1136/ bmjopen-2020-038128

- Prepublication history for this paper is available online. To view these files, please visit the journal online (http://dx.doi. org/10.1136/bmjopen-2020038128).

Received 02 March 2020 Revised 14 May 2020 Accepted 23 June 2020

D) Check for updates

(c) Author(s) (or their employer(s)) 2020. Re-use permitted under CC BY-NC. No commercial re-use. See rights and permissions. Published by BMJ.

${ }^{1}$ Faculty of Medicine, University of Cyberjaya, Persiaran Bestari, Cyberjaya, Selangor, Malaysia 2Department of Pharmacology, Faculty of Medicine, University of Malaya, Kuala Lumpur, Malaysia

Correspondence to Associate Professor Dr. Mohammed Abdullah Alshawsh; alshaweshmam@um.edu.my

\section{ABSTRACT}

Introduction Colorectal cancer is one of the leading causes of cancer-related morbidity worldwide and it has been reported to be associated with poor lifestyle habits which include excess tobacco and alcohol intake as well as genetics and age factors. Probiotics such as the lactic acid bacteria and Bifidobacterium as well as probiotic containing foods (kombucha, kefir, miso etc) have received lots of attention as anticancer agents for prevention and treatment. The effects of the administration of probiotics to patients with colorectal cancer is the primary goal of this systematic review. The overall aim is to assess how the use of probiotics in patients with colorectal cancer helps in the management of colorectal cancer and its effect on the diversity of gut microbiota. The final systematic review will provide a comprehensive evidence base for the use and efficacy of probiotics in patient with colorectal cancer care. Methods and analysis The systematic review, will be conducted by extensively searching different databases such as PubMed, Web of Science, Scopus, Wiley and ProQuest to identify randomised controlled trials (with no time frame) which relate to the administration of probiotics to patients with colorectal cancer. The search strategy will include words like colorectal cancer, probiotics, Bifidobacterium, clinical trials etc. A systematic search of databases was performed between 17 and 20 January 2020. Two reviewers will independently review the studies and also search the reference lists of the eligible studies to obtain more references. Data will be extracted from the eligible studies using standardised data extraction form. After assessing the risk of bias, qualitative analysis will be used to synthesise the systematic review.

Ethics and dissemination This is a protocol for a systematic review; therefore, it doesn't require any ethics approval. We intend to disseminate the protocol in a peer reviewed journal.

\section{INTRODUCTION}

Colorectal cancer (CRG) refers to tumours that start in the colon and spreads all the way to the rectum. Different types of colorectal polyps exist, but CRC usually develops from adenomas. CRC is one of the very common causes of mortality among patients with cancer worldwide including developed and
Strengths and limitations of this study

- The findings from this systematic review will provide current insight on how probiotics are used either alone or in combination to improve the quality of life of patients with colorectal cancer.

- This study will highlight the efficacy and the underlying mechanism of actions of probiotics against colorectal cancer.

- The findings from this review will improve our knowledge of the beneficial effects of probiotics in prevention and management of colorectal cancer.

- There will be limitations inherent to any systematic review such as the lack of information on outcome variables, as well as the assumption that the evaluation techniques are consistent across studies.

- Only studies in English language will be included in this systematic review.

undeveloped countries but mostly in first world countries. It is predicted that by 2035 , over 25 million incidences of CRC will be discovered on a yearly basis. ${ }^{1}$ It is also estimated that over 376000 new cases of CRC diagnosis as well as approximately 200000 deaths take place yearly in China. ${ }^{2}$

CRC proves to be a silent killer ailment that may not be noticed in time until the cancer has progressed significantly. Symptoms of CRC resemble symptoms of several ailments and is easily misdiagnosed unless a colonoscopy is done. The symptoms of CRC include unexplained anaemia, unexplained weight loss, bloating, changes in the bowel movement habits, bloody stool, vomiting and pelvic pain. It has been proven that the initiating events of CRC include TP53 mutation in CRC associated with colitis as well as mutation in sporadic CRC. ${ }^{3}$ Different causes of CRC have been examined over the years from data collected in cohort-based studies and these findings resemble studies carried out in 
animal models, The common conclusion is that age, lifestyle choices such as smoking and excessive alcohol intake which can lead to obesity or diabetes, as well as genetic risk factors, contribute to the development of CRC. ${ }^{45}$

CRC can also be inherited through the genes by inheriting mutated genes that trigger tumour growth, but this only accounts for about $5 \%$ of CRC cases. ${ }^{6}$ In addition, different researchers in their studies have agreed that an increased number of opportunistic bacteria which quickly turn pathogenic, such as Helicobacter pylori, Bacteroides fragilis, Helicobacter hepaticus, enterotoxigenic Escherichia coli, Fusobacterium nucleatum and Streptococcus bovis, can lead to the initiation of adenomas formation that lead to CRC. ${ }^{7}$

Patients with CRC usually undergo surgery to remove cancerous polyps or to remove some part of their colon which have been affected (colon resection). Others undergo chemotherapy or radiotherapy to treat CRC. These treatment options are sometimes unsuccessful or lead to a myriad of severe side effects which increase hospital stay time and sometimes morbidity. ${ }^{8}$

Probiotics is redefined by the International Scientific Association for Probiotics and Prebiotics (ISAPP) as 'live microorganisms which when administered in adequate amounts, confer a health benefit on the host' ${ }^{9}$ Probiotic microorganisms are special because they are capable of surviving in the human gastrointestinal tract before they get to the colon, where the majority of their metabolic activity is carried out. They include lactic acid producing bacteria of the genera Lactobacillus and Bifidobacterium as well as Propionibacterium, Saccharomyces and are the major ingredients in yoghurts and other functional foods such as unfermented milks, cheese, kefir and fermented milk. ${ }^{10}$

On the other hand, prebiotics are usually termed as non-digestible carbohydrates such as inulin and oligosaccharides, soy and resistant starch. Prebiotics is defined by ISAPP as 'a substrate that is selectively used by host microorganisms to confer health benefit to the host'. ${ }^{9}$ Prebiotics stimulate an increased growth of probiotics by providing a more favourable environment for their growth. ${ }^{11}$ Leading to a gut environment that promotes the competitive dismissal of opportunistic and potentially pathogenic bacteria which could initiate the beginning of CRC. ${ }^{11}$ Several studies have shown that the administration of both probiotics and prebiotics as a combination can aid increasingly in improving the conditions of patients with CRC especially after colorectal surgery has been performed. ${ }^{12} 13$

Probiotics have been used by the traditional healers for the prevention and treatment of different types of illneses from the simple stomach ache to intestinal neoplasia. In addition various experimental studies have shown that continious ingestion of probiotic bacteria can enhance the qualitative as well as quantitative components of the gut microbiota. ${ }^{14}$ In one instance, the ingestion of Lactobacillus acidophilus LA-11, L. plantarum CGMCC 1258 and Bifidobacterium longum BL-88 $\left(2.6 \times 10^{14}(\mathrm{CFU}) /\right.$ day $)$ for 16 days, resulted in an increase in the diversification of gut microflora and microbial richness in patients suffering from CRC who have been scheduled for colorectomy. Eventually, the microbial flora makeup of these individuals improved to resemble that of individuals without CRC. ${ }^{15}$ Probiotic bacteria when consumed in adequate quantittes are able to diminish the total quantity of non beneficial disease causing bacteria found in the colon by numerous mechanisms, particulary as regards; rivalry for nutrients, growth factors, and adhesion of the probiotics onto the intestinal cells of the host. ${ }^{16}$ Ingestion of probiotic also inhibits the activity of pathobionts such as Clostridium perfringens and Klebsiella pneumonia which are potential pathogenic microorganisms and could also be symbiotic microorganisms under certain gut environment conditions. ${ }^{17}$ Some probiotic bacteria can produce antibacterial substances such as bacteriocins, hydrogen peroxide, lactic acid and reuterin, which decrease the growth or totally eradicate pathogenic bacteria from the colon. The very popular advantages of the consumption and use of probiotics in the management and treatment of diarrhoea associated to anti-cancer chemotherapy revolves around the restoration to normal of the intestinal microbiota. ${ }^{18}$ The favourable altreation by probiotic bacteria in the makeup of the gut microbiome is closely associated with the reduced risk of suffering from CRC in the future. ${ }^{19}$ Production of short chain fatty acids by probiotics which leads to cell apoptosis is one of the aways through which probiotics reduce the proliferation of colorectal carcinaoma. ${ }^{20}$ Scientific eveidence by various in-vitro and in-vivo studies have concluded that various strains of probiotics possess anti-carcinogenic properties via different mechanisms. ${ }^{21} 22$

Based on our search on systematic reviews related to our topic, we found out that most of the systematic reviews which have been done were not entirely specific to $\mathrm{CRC},{ }^{12}$ and those that are specific to probiotics and patients with CRC focus on one outcome either on postoperative complications, ${ }^{23}$ surgical site infection, ${ }^{24}$ diarrhoea from chemotherapy. ${ }^{25}$ We see this as a limitation of these studies, hence we intend to study more than one outcome in order to get a holisitc idea of how probiotics administration affect patients with CRC who are receiving different types of treatment on different levels. As we will asses several outcomes, these outcomes will be categorised and discussed based on if they are primary or secondary outcomes. Previously published reviews related to probiotics mostly investigated its effect on CRC and the mechanisms through which probiotics ameliorate CRC using diverse models including pre-clinical studies, and in-vitro studies. ${ }^{26}{ }^{27}$ Some reviews also focused more on the use of specific probiotic as anticancer adjuvant. ${ }^{28}$ Our systematic review is unique and different from other reviews in which we intend to include only randomised clinical trial studies (RCT) and asses the effects of the adminstration of various types of probiotics on patients with CRC. 


\section{Review aim}

To systematically review, assess and summarise and interpret clinical trials studies on how the use of probiotics compared with placebo in patients with CRC in helps in the treatment, and management of CRC. In addition, this study will critically summarise how probiotics administration in patients with CRC affect the diversity of gut microbiome and patient quality of life.

\section{METHODS AND ANALYSIS}

This systematic review protocol goes in accordance to the Preferred Reporting Items for Systematic Review and Meta-Analysis Protocols (PRISMA-P) guidelines. ${ }^{29}$

\section{Eligibility criteria for included studies}

The inclusion criteria include studies on patients with CRC who are were treated with chemotherapy, radiotherapy or surgery. The included studies must be carried out as randomised controlled trials with either a comparator group, control group or placebo group. Details of the inclusion and exclusion criteria that will be used in the systematic review is in table 1 .

\section{Search strategy}

The relevant studies will be identified using standard search terms for individual databases. Randomised controlled trials will be identified from PubMed, Web of Science, Scopus, Wiley and ProQuest. The search results will be filtered to identify studies only in English. The bibliography of all included randomised controlled trials will be reviewed to identify any trials missed during the initial database search. This will be done independently by two reviewers. Search terms will be used and connected by Boolean AND/OR operators:

\section{Table 1 Eligibility criteria based on PICOS model}

\section{Items based on PICOS} model

1. Population or participants and conditions of interest

\section{Eligibility criteria}

Humans, any age, diagnosed with colorectal cancer (CRC) or with colon cancer or rectal cancer and have been treated with probiotics as an intervention and this will include:

Patients with CRC who have had colorectal surgery or colon resection or haven't had surgery. Patients with CRC who had or are still undergoing chemotherapy or radiotherapy or not.

2. Interventions or exposures

Probiotics of any kind (eg, Lactobacillus, Bifidobacterium, Propionibacterium, Saccharomyces, etc) used on its own or in combinationwith other probiotics or combination with prebiotics such as inulin orresistant starch.

\section{Comparisons or control groups}

4. Outcomes of interest

\section{Placebos, or healthy people of any age, without CRC.} Baseline comparison of patients before the intervention.

\section{Primary outcomes}

- Effects of probiotics on the diversity of human gut microbiota.

- Effects of probiotics on inflammatory biomarkers relevant to CRC.

- Immunoregulatory action of probiotics.

Secondary outcomes

- Patient status (improvement/ no improvement of colorectal carcinoma) after administration of probiotics.

- Prognosis such as imaging to compare the size of cancer tumour before and after intervention.

- General health and improvement in quality of life of the patient.

Adverse events such as morbidity and mortality.

$\begin{array}{ll}\text { 5. Study designs } & \text { Clinical trials, randomised clinical trials. } \\ \text { 6. Other exclusion criteria } & \text { Articles not in English language. } \\ & \text { Reviews. } \\ & \text { Animal or in vitro work done with zprobiotics. } \\ & \text { Studies not about CRC or rectal or colon cancer. } \\ & \text { Studies not testing the role of probiotics on CRC or rectal or colon cancer patients. }\end{array}$

PICOS model, P - Patient, Problem or Population

I - Intervention

C - Comparison, control or comparator

O - Outcome of interest

S - Study type. 
The search syntax for PubMed will include:

1. Probiotic* OR Lactobacillus OR Bifidobacterium OR Propionibacterium OR Saccharomyces OR "Bacillus coagulans".

2. colon OR colorectal OR colonic OR rectal.

3. cancer OR neopla* OR tumo* OR carcinoma OR malignan*.

4. clinical trial OR trial* OR "intervention study" OR RCT OR "randomized controlled trial" OR "randomised controlled trial".

5. \#2 AND \#3.

6. \#1 AND \#4 AND \#5.

The search syntax for other databases will be using similar approach as PubMed or using the following merged search terms:

(Probiotic OR Lactobacillus OR Bifidobacterium OR Propionibacterium OR Saccharomyces OR "Bacillus coagulans") AND (colon OR colorectal OR colonic OR rectal) AND (cancer OR neopla* OR tumo* OR carcinoma OR malignan*) AND ("clinical trial" OR "intervention study" OR "RCT" OR "randomised controlled trial" OR "randomized controlled trial")

A systematic search of PubMed, Scopus, Web of Science, ProQuest and Wiley online library was performed between 17 and 20 January 2020.

\section{Selection process of included studies}

The primary article screening will be carried out independently by two reviewers. Titles and abstract of the studies will be screened independently, and the selected studies will be divided into three groups: relevant, irrelevant and unsure. The studies which are categorised as irrelevant by both reviewers will then be eliminated from the review. The full text of the remaining studies will be then reviewed by both reviewers using the eligibility criteria and studies that meet all the criteria will be included. In case of discrepancy, the two reviewers will first meet to discuss their choices and a final decision will be made. If there is any misunderstanding or conflict a third opinion will be sought from the other reviewers and when an agreement is reached, a final decision will be made.

\section{Data extraction and analysis}

Studies meeting the inclusion criteria will be processed for data extraction. Two authors (IJD and MAA) will independently screen title and abstract, and then full text. The data will be extracted and recorded in a consistent way using standardised data extraction form. The following data will be extracted: study year, author/s, study title, number of participants, stage of CRC, type of probiotic used, dosage of intervention, duration of intervention, control or placebo used, primary outcomes, secondary outcomes, conclusion and limitation.

The search and study framework will be represented using PRISMA flow chart ${ }^{30}$ and the numbers of all included and excluded studies will be reported and the reasons for exclusion of studies will be given.

\section{Assessment of risk of bias of included studies}

The risk of bias will be assessed according to the guidelines of the Cochrane Collaboration using ROB tool. ${ }^{31}$ This tool will be based on the following domains: random sequence generation, allocation concealment, adequacy of blinding for participants, blinding of outcome assessment, incomplete outcome data and selective reporting and other sources of bias. RoB 2.0 will be used for risk of bias assessment of included study via RevMan V.5.3 software. ${ }^{32}$ Two reviewers independently will carry out the assessment and if there is any conflict, third opinion will be obtained from third partner.

\section{Strategy for data synthesis}

Initial screening of the relevant RCT studies showed that most of the outcomes of the included studies are not homogenous and cannot be pooled together, therefore meta-analysis most likely will not be carried out. Instead, a qualitative analysis will be performed to synthesise the studies included in the systematic review as well as a critical appraisal of the outcomes will be considered for all studies. However, after we complete the data extraction of all included studies if we find out that any of the outcomes is homogenous across some of the studies, then a meta-analysis of those selected outcomes will be carried out. The quality of all included studies will be assessed by using PRISMA checklist ${ }^{30}$ to ensure that the included studies are of good quality and to ensure that there is no publication bias.

\section{Patients and public involvement}

There will be no need to involve patients or members of the general public in the design of this systematic review, and no patients or member of the public will be contacted in order to complete the systematic review.

\section{Ethics and dissemination}

Findings of this systematic review will be published in a peer-reviewed publication and will be presented at a professional conference. Because this is only a protocol, no ethical assessment is required.

Contributors IJD and MAA contributed to the conception of the study. The systematic review protocol was drafted by IJD and was reviewed by MAA and AMA-K. The search strategy was developed by IJD and MAA and will be performed by IJD and MAA, who will also independently screen the potential studies, extract data from the included studies, assess the risk of bias, and complete the data synthesis. AMA-K and SH will arbitrate in cases of disagreement and ensure the absence of errors. All authors reviewed and approved the publication of the protocol.

Funding This study was supported by a research grant from University of Malaya, project number (ST015-2020).

Competing interests None declared.

Patient and public involvement Patients and/or the public were not involved in the design, or conduct, or reporting, or dissemination plans of this research.

Patient consent for publication Not required.

Provenance and peer review Not commissioned; externally peer reviewed.

Open access This is an open access article distributed in accordance with the Creative Commons Attribution Non Commercial (CC BY-NC 4.0) license, which permits others to distribute, remix, adapt, build upon this work non-commercially, 
and license their derivative works on different terms, provided the original work is properly cited, appropriate credit is given, any changes made indicated, and the use is non-commercial. See: http://creativecommons.org/licenses/by-nc/4.0/.

\section{ORCID iDs}

Ifeoma Julieth Dikeocha http://orcid.org/0000-0003-1700-828X

Mohammed Abdullah Alshawsh http://orcid.org/0000-0001-8342-5183

\section{REFERENCES}

1 Dos Reis SA, da Conceição LL, Siqueira NP, et al. Review of the mechanisms of probiotic actions in the prevention of colorectal cancer. Nutr Res 2017;37:1-19.

2 Chen W, Zheng R, Baade PD, et al. Cancer statistics in China, 2015. CA Cancer J Clin 2016;66:115-32.

3 Charbonneau MR, Blanton LV, DiGiulio DB, et al. A microbial perspective of human developmental biology. Nature 2016:535:48-55.

4 Shao T, Yang Y-X. Cholecystectomy and the risk of colorectal cancer. Am J Gastroenterol 2005;100:1813-20.

5 Song M, Chan AT, Factors E. Gut microbiota, and colorectal cancer prevention. Clin Gastroenterol Hepatol 2019;17:275-89.

6 Mármol I, Sánchez-de-Diego C, Pradilla Dieste A, et al. Colorectal carcinoma: a general overview and future perspectives in colorectal cancer. Int J Mol Sci 2017;18:197.

7 Ai L, Tian H, Chen Z, et al. Systematic evaluation of supervised classifiers for fecal Microbiota-Based prediction of colorectal cancer. Oncotarget 2017;8:9546-56.

8 Darbandi A, Mirshekar M, Shariati A, et al. The effects of probiotics on reducing the colorectal cancer surgery complications: a periodic review during 2007-2017. Clin Nutr 2019;S0261-5614:33135-8.

9 Gibson GR, Hutkins R, Sanders ME, et al. Expert consensus document: the International scientific association for probiotics and prebiotics (ISAPP) consensus statement on the definition and scope of prebiotics. Nat Rev Gastroenterol Hepatol 2017;14:491-502.

10 Uccello M, Malaguarnera G, Basile F, et al. Potential role of probiotics on colorectal cancer prevention. BMC Surg 2012;12:S35.

11 Roberfroid M. Prebiotics: the concept revisited. J Nutr 2007;137:830S-7.

12 Lytvyn L, Quach K, Banfield L, et al. Probiotics and synbiotics for the prevention of postoperative infections following abdominal surgery: a systematic review and meta-analysis of randomized controlled trials. $J$ Hosp Infect 2016;92:130-9.

13 Kasatpibal N, Whitney JD, Saokaew S, et al. Effectiveness of probiotic, prebiotic, and synbiotic therapies in reducing postoperative complications: a systematic review and network meta-analysis. Clin Infect Dis 2017;64:S153-60.

14 Chang J-H, Shim YY, Cha S-K, et al. Effect of Lactobacillus acidophilus KFRI342 on the development of chemically induced precancerous growths in the rat colon. J Med Microbiol 2012;61:361-8.

15 Liu Z, Qin H, Yang Z, et al. Randomised clinical trial: the effects of perioperative probiotic treatment on barrier function and post- operative infectious complications in colorectal cancer surgery - a double-blind study. Aliment Pharmacol Ther 2011;33:50-63.

$16 \mathrm{Ku} \mathrm{S}$, Park MS, Ji GE, et al. Review on Bifidobacterium bifidum bgn4: functionality and nutraceutical applications as a probiotic microorganism. Int J Mol Sci 2016;17:1544.

17 Vitetta L, Vitetta G, Hall S. Immunological tolerance and function: associations between intestinal bacteria, probiotics, prebiotics, and phages. Front Immunol 2018;9:2240.

18 Gamallat Y, Meyiah A, Kuugbee ED, et al. Lactobacillus rhamnosus induced epithelial cell apoptosis, ameliorates inflammation and prevents colon cancer development in an animal model. Biomed Pharmacother 2016;83:536-41.

19 Serban DE. Gastrointestinal cancers: influence of gut microbiota, probiotics and prebiotics. Cancer Lett 2014;345:258-70.

20 Fotiadis C-I, Stoidis C-N, Spyropoulos B-G, et al. Role of probiotics, prebiotics and synbiotics in chemoprevention for colorectal cancer. World J Gastroenterol 2008;14:6453-7.

21 de Ameida Brasiel PG, Luquetti S, MdCG P, et al. Preclinical evidence of probiotics in colorectal carcinogenesis: a systematic review. Dig Dis Sci 2020:1-14.

22 Eslami M, Yousefi B, Kokhaei P, et al. Importance of probiotics in the prevention and treatment of colorectal cancer. J Cell Physiol 2019;234:17127-43.

23 Liu PC, Yan YK, Ma YJ, et al. Probiotics reduce postoperative infections in patients undergoing colorectal surgery: a systematic review and meta-analysis. Gastroenterol Res Pract 2017;2017:1-9.

24 Skonieczna-Żydecka K, Kaczmarczyk M, Łoniewski I, et al. A systematic review, meta-analysis, and meta-regression evaluating the efficacy and mechanisms of action of probiotics and synbiotics in the prevention of surgical site infections and surgery-related complications. J Clin Med 2018;7:556.

25 Lu D, Yan J, Liu F, et al. Probiotics in preventing and treating chemotherapy-induced diarrhea: a meta-analysis. Asia Pac J Clin Nutr 2019;28:701-10.

26 Cruz BCS, Sarandy MM, Messias AC, et al. Preclinical and clinical relevance of probiotics and synbiotics in colorectal carcinogenesis: a systematic review. Nutr Rev 2020:nuz087.

27 Sivamaruthi BS, Kesika P, Chaiyasut C. The role of probiotics in colorectal cancer management. Evid Based Complement Alternat Med 2020;2020:1-17.

28 Vivarelli S, Falzone L, Basile M, et al. Benefits of using probiotics as adjuvants in anticancer therapy (review). World Acad Sci J 2019;1:125-35

29 Shamseer L, Moher D, Clarke M, et al. Preferred reporting items for systematic review and meta-analysis protocols (PRISMA-P) 2015 elaboration and explanation. BMJ 2015;349:g7647.

30 Moher D, Liberati A, Tetzlaff J, et al. Preferred reporting items for systematic reviews and meta-analyses: the PRISMA statement. Ann Intern Med 2009;151:264-9.

31 Sterne JAC, Savović J, Page MJ, et al. RoB 2: a revised tool for assessing risk of bias in randomised trials. BMJ 2019;366:14898.

32 Cochrane Centre. Review Manager (RevMan)[Computer Program] Version 5.2. 3. Copenhagen: The Nordic Cochrane Centre; 2012. Health Psychology Review 2014;17. 